\title{
Widefield imaging with Clarus fundus camera vs slit lamp fundus examination in assessing patients referred from the National Health Service diabetic retinopathy screening programme
}

\author{
Wei Sing Lim $\mathbb{D}^{1} \cdot$ Gabriela Grimaldi $^{1} \cdot$ Luke Nicholson $^{1} \cdot$ Khadijah Basheer $^{1} \cdot$ Ranjan Rajendram $^{1}$
}

Received: 10 June 2020 / Revised: 29 September 2020 / Accepted: 2 October 2020 / Published online: 20 October 2020

(c) The Author(s), under exclusive licence to The Royal College of Ophthalmologists 2020

\begin{abstract}
Objectives To compare diabetic retinopathy (DR) grading and management plan between virtual review using widefield Clarus imaging and macular optical coherence tomography (OCT) versus slit lamp clinical examination and macular OCT. Method New referrals over 3 months from the National Diabetic Eye Screening programme (DESP) were screened. Patients who had both Clarus widefield imaging and macular OCT were included. All patients underwent slit lamp examination in clinic. Data obtained from electronic patient records included referral reason, DR grading and management plan. Two graders retrospectively reviewed imaging and formulated a management plan blinded to results from patients' clinic visit. Results from virtual examination were compared with those from slit lamp examination.

Results One-hundred and two eyes of 51 patients were assessed. 11 fundus photos from 7 patients and 15 fundus photos from 10 patients were deemed inadequate by grader G1 and G2, respectively. Eighteen (35\%) patients and $11(22 \%)$ patients from virtual assessment by G1 and G2, respectively were found to need a face a face appointment to aid diagnosis. Compared to slit lamp examination, $15 \%$ and $7.5 \%$ of patients from G1 and G2's virtual assessment respectively had different proposed management plan. Agreement of DR grading between both virtual graders and slit lamp examination was fair (Kappa's coefficient $=0.56$ ). One case of slit lamp noted retinal neovascularization, which was graded as background retinopathy by DESP was also graded as such on virtual assessment.

Conclusion Widefield Clarus and OCT imaging allowed two-thirds of DESP referrals to be safely managed virtually.
\end{abstract}

\section{Introduction}

The National Health Service (NHS) Diabetic Eye Screening Programme (DESP) in UK has been operational since 2003 [1]. It offers annual screening with fundus photographs to all diabetic patients aged 12 or over. In England, two fundus photographs of $45^{\circ}$ each are used per eye, one centred on the fovea and the other centred on the disc. This will give a combined field of about $60^{\circ}$ wide. If the fundus photographs show any pathology that might require monitoring or treatment by a specialist, or if the photographs are of inadequate quality, then the patient will be referred to the hospital eye clinic for further management.

Wei Sing Lim

weising.lim@nhs.net

1 Moorfields Eye Hospital NHS Foundation Trust, London, UK
The prevalence of diabetes in UK and the world is expected to grow further due to ageing and higher rates of obesity [2]. The Royal College of Ophthalmologists anticipated a $60 \%$ increase in the diabetic retinopathy (DR) case load over the next 20 years. It published 'The Way Forward' [3] documents in 2015 to identify new methods and ways of working to meet the increasing demand for ophthalmic services. Specifically, it recommended using virtual clinics to follow up stable moderate non proliferative diabetic retinopathy if sufficient retinal coverage can be achieved at adequate quality by the available imaging modalities. Other patients that can be moved into a virtual clinic service include those who have been stable off treatment for 3 months or more. Other non-doctor, allied health care professionals can be trained to review the images within the virtual service rather than a senior ophthalmologist reviewing all images.

The use of virtual clinics is becoming widespread in UK today [4-6]. In these clinics, patients do not see a doctor face to face, rather their fundus photographs are reviewed 
by a doctor or grader to decide on further management. The use of virtual clinics has allowed a higher volume of patients to be seen in a healthcare setting with constrained clinic space and limited staffing levels. Indeed 'The Way Forward' document identified that many patients with diabetes do not require intervention. The use of virtual clinics was shown to reduce patients' waiting time in clinic, possibly leading to better compliance with follow up clinic visits [7]. It must be remembered that diabetes may affect multiple systems, and patients may be attending those specialist clinics as well as the eye clinic, and people with diabetes are often of working age and therefore efficient throughput of their eye services is important.

Most virtual clinics in UK at present employ the Optos fundus camera (Optos PLC, Dunfermline, Scotland, UK). The Zeiss Clarus fundus camera (CLARUS 500"T , Carl Zeiss Meditec AG, Jena, Germany) has been introduced to UK relatively recently in 2018 .

Previous studies have shown that examination with Optos ultra-widefield fundus imaging is reliable in detecting diabetic retinopathy and compares well to slit lamp fundus examination, yielding a sensitivity and specificity of $73 \%$ and $96 \%$, respectively for detecting proliferative diabetic retinopathy [8-11]. However no studies have been published comparing Clarus widefield fundus camera with slit lamp fundus examination.

This study's main objective was to assess the validity and safety of widefield Clarus fundus imaging for new DESP(DR and non-DR) referrals, comparing outcomes from virtual review using widefield Clarus fundus imaging and macular OCT with the Standard of Care outpatient clinic assessment with slit lamp examination and Macular OCT. Secondary objectives were to measure the agreement in DR grading between slit lamp and virtual assessment and to analyse intergrader reliability.

\section{Methods}

This was a multisite retrospective clinic-based study conducted at Moorfields Eye Hospital, London, United Kingdom. Approval for retrospective anonymized image analysis from the institutional review board of Moorfields Eye Hospital was obtained and the study was conducted in accordance with the tenets of the Declaration of Helsinki.

A list of all new DESP referrals to the hospital eye service at Moorfields St Georges and Moorfields City Road from 13th May 2019 to 8th August 2019 was screened. The author WL then engaged the help of other fellows to request for Clarus fundus imaging when those patients attended their clinic appointment. There was no selection bias for patient with more or less pathology. We included only those patients who had Clarus widefield fundus photographs in addition to Topcon 3D macula $6 \times 6 \mathrm{~mm}$ OCT scans
(Topcon 3D OCT-2000, Topcon, Tokyo, Japan). All patients had been seen by an ophthalmologist who carried out slit lamp fundus examination in a Medical Retina clinic.

The OCT would be used to form the management plan in the clinic and was included in the virtual assessment to allow similar support for the virtual derived management plan. Data concerning the outcome of face-to-face slit lamp examination in the eye clinic were obtained from electronic patient records, including referral reason, diabetic retinopathy grading, presence of other retinal pathology and final management plan.

The two graders WL and GG who are medical retina fellows and also authors of this paper retrospectively reviewed the patients' Clarus fundus photographs and OCT scans blinded to the outcome of face-to-face (standard care) clinical examination, assessing DR grading and clarity of the fundus photographs, recording the presence of other retinal pathology and providing a management plan. Graders were also required to assess if the available imaging was adequate to formulate a diagnosis and management plan for each patient without face-to-face review.

The diagnosis and management plan for those patients whose imaging was deemed adequate by the graders were then compared with those obtained from face-to-face slit lamp assessment.

Diabetic retinopathy was graded according to the English Diabetic Eye Screening Programme Grading Classification adapted from the NHS diabetic eye screening programme [1]:

R0 no diabetic retinopathy

R1 background diabetic retinopathy

$\mathrm{R} 2$ moderate to severe proliferative diabetic retinopathy

R3A active proliferative diabetic retinopathy

R3S stable proliferative diabetic retinopathy

M0 No maculopathy

M1 Maculopathy present

To assess agreement between the virtual and slit-lamp DR grading and the intergrader reliability, Cohen's Kappa coefficient was calculated using Prism 8 (Graphpad software, San Diego, CA). Guidelines for interpretation of Kappa coefficient based on Landis and Koch's paper [12] are as follow:

$0-0.2$ slight agreement

$0.21-0.4$ fair agreement

$0.41-0.6$ moderate agreement

$0.61-0.8$ substantial agreement

$0.81-1.00$ almost perfect agreement

\section{Results}

\section{Overall data}

One hundred and two eyes from 51 consecutive patients were included in this study. All 51 patients were referred 
Table 1 Reason for referral from Diabetic Eye Screening Programme.

\begin{tabular}{ll}
\hline Referred pathology & Number of eyes \\
\hline R2M1 & 22 \\
R1M1 & 20 \\
R2M0 & 10 \\
R3SM1 & 1 \\
R3A & 5 \\
Cataract & 7 \\
Branch retinal vein occlusion & 5 \\
Age related macular degeneration & 4 \\
Ungradable & 4 \\
Unexplained vision drop & 2 \\
Choroidal naevus & 1 \\
Coats disease & 1 \\
Epiretinal membrane & 1 \\
Macroaneurysm & 1 \\
Total & 84 \\
\hline
\end{tabular}

from DESP because at least one of their eyes had retinal pathology that might potentially require treatment.

The breakdown of retinal pathologies in individual eyes from the referral letter is as follow in Table 1:

Eighty-four eyes from 51 consecutive patients in our study had pathology referred for possible treatment and the remaining 18 eyes from 18 patients did not have referable pathology.

All patients had a referral letter from the DESP. Two eyes did not have Clarus fundus photos likely because of media opacity recorded in clinical notes. Out of the 100 eyes with Clarus photos, 86 eyes had montage of two photos (Ultra-widefield) and 14 eyes only had single photo (Widefield). All eyes except for one had macula OCT.

From the assessment by G1, 11 fundus photos (11\% of all photos) from 7 patients were deemed inadequate. From the assessment by G2, 15 fundus photos (15\% of all photos) from 10 patients were deemed inadequate.

Out of the 16 eyes with other non-diabetic retinopathy retina pathology, there was $81 \%$ agreement (13 out of 16 eyes) in diagnosis between virtual grader G1 and clinical examination. In comparison there was an 88\% (14 out of 16 eyes) agreement between grader G2 and clinical examination.

From the virtual assessment by G1, 33 patients (65\%) were deemed to have adequate imaging to enable diagnosis and management plan without seeing the patient face to face. For virtual grader G2, 40 patients (78\%) were deemed to have adequate imaging.

There was one patient who had photic maculopathy. The retinal images were of adequate quality but both grader G1 and G2 were not able to diagnose photic maculopathy without speaking to the patient to take an essential history.
One eye was diagnosed as having branch retinal vein occlusion by DESP and virtual grader G1 but not by clinical examination or virtual grader $\mathrm{G} 2$.

\section{Comparing management plan between virtual examination and clinical examination}

Table 2 below compares the management plan from clinical examination with that of virtual grader 1 (A), between clinical examination and virtual grader 2 (B) and lastly between virtual grader 1 and virtual grader 2 (C).

Virtual diagnoses that were made included non proliferative or stable treated proliferative diabetic retinopathy, branch retinal vein occlusion, macular degeneration, Coats disease, macroaneurysm and vitreomacular traction.

Eighteen $(35 \%)$ patients and $11(22 \%)$ patients from virtual grading by $\mathrm{G} 1$ and $\mathrm{G} 2$, respectively were found to need a face a face appointment to obtain further information to aid diagnosis. Reasons for this were poor fundus view, reduced vision that could not be explained fully by imaging, active retina neovascularization and choroidal naevus.

Out of the 33 patients whom G1 decided could be managed without face to face examination, five patients were treated differently from the face to face clinic management plan. In the face to face clinic, three of them had fluorescein angiogram carried out. Out of those three, one was found to have diabetic macular oedema requiring laser, one was found to have severe non proliferative diabetic retinopathy and the remaining one was found to have early stage proliferative diabetic retinopathy on fluorescein angiography, which was not definite and atypical on the clinical examination.

The decision for focal macula laser was likely due to variation in management plan of different doctors rather than because of fluorescein angiogram findings. Fluorescein angiography is immediately available in the standard outpatient clinic and the ease of access and the wish to obtain a baseline fluorescein may have led to this request.

One patient was assessed to need Intravitreal anti-VEGF for diabetic macula oedema by grader G1 but not by the doctor who saw the patient face to face. One patient with vitreomacular traction was referred to vitreoretinal team by the clinic doctor but was planned for observation by G1. It is likely the patient's symptoms contributed to the doctor's decision to refer the patient to vitreoretinal team. The patient was seen by the vitreoretinal surgical team and did not require vitreoretinal surgery.

Out of the 40 patients whom G2 decided could be managed without face to face examination, three patients were treated differently from the face to face clinic. Two patients had fluorescein angiogram carried out when they were seen face to face. One patient was assessed by the virtual grader to need intravitreal anti-VEGF for diabetic 
Table 2 (A) Comparing management plan from clinical examination with that of virtual grader 1. (B) Comparing management plan from clinical examination with that of virtual grader 2. (C) Comparing management plan between virtual grader 1 and virtual grader 2 .

\begin{tabular}{|c|c|c|c|c|c|c|c|c|c|c|}
\hline \multicolumn{11}{|l|}{$\mathrm{A}$} \\
\hline \multicolumn{11}{|c|}{ Virtual grading by $\mathrm{G} 1$} \\
\hline & \multicolumn{2}{|c|}{ Need face to face review } & \multicolumn{2}{|l|}{ Discharge } & \multicolumn{2}{|c|}{ Observe } & \multicolumn{2}{|c|}{ More tests } & Treatment & Total \\
\hline \multicolumn{11}{|c|}{ Clinical examination } \\
\hline Discharge & \multicolumn{2}{|l|}{2} & \multicolumn{2}{|l|}{0} & \multicolumn{2}{|l|}{0} & \multicolumn{2}{|l|}{0} & 0 & 2 \\
\hline Observe & \multicolumn{2}{|l|}{8} & \multicolumn{2}{|l|}{2} & \multicolumn{2}{|l|}{18} & \multicolumn{2}{|l|}{1} & 1 & 30 \\
\hline More tests & \multicolumn{2}{|l|}{2} & \multicolumn{2}{|l|}{0} & \multicolumn{2}{|l|}{4} & \multicolumn{2}{|l|}{5} & 0 & 11 \\
\hline Treatment & \multicolumn{2}{|l|}{6} & \multicolumn{2}{|l|}{0} & \multicolumn{2}{|l|}{0} & \multicolumn{2}{|l|}{0} & 2 & 8 \\
\hline \multicolumn{11}{|l|}{ B } \\
\hline \multicolumn{11}{|c|}{ Virtual grading by G2 } \\
\hline & \multicolumn{2}{|c|}{ Need face to face review } & Discharge & & Obsery & & Mor & e tests & Treatment & Total \\
\hline Clinical ex & ination & & & & & & & & & \\
\hline Discharge & 1 & & 0 & & 1 & & 0 & & 0 & 2 \\
\hline Observe & 4 & & 8 & & 15 & & 2 & & 1 & 30 \\
\hline More tests & 1 & & 0 & & 2 & & 7 & & 1 & 11 \\
\hline Treatment & 5 & & 0 & & 0 & & 0 & & 3 & 8 \\
\hline $\mathrm{C}$ & & & & & & & & & & \\
\hline Virtual gra & ig by $\mathrm{G} 2$ & & & & & & & & & \\
\hline & & Need face to & e review & & charge & & erve & More tests & s Treatment & Total \\
\hline Virtual gra & g by G1 & & & & & & & & & \\
\hline Need face & face review & 11 & & 1 & & 3 & & 2 & 1 & 18 \\
\hline Discharge & & 0 & & 2 & & 0 & & 0 & 0 & 2 \\
\hline Observe & & 0 & & 5 & & 14 & & 2 & 1 & 22 \\
\hline More tests & & 0 & & 0 & & 1 & & 5 & 0 & 6 \\
\hline Treatment & & 0 & & 0 & & 0 & & 0 & 3 & 3 \\
\hline
\end{tabular}

macula oedema but not by the doctor who saw the patient face to face.

\section{Comparing diabetic retinopathy grading between virtual examination and clinical examination}

Table 3 shows how the diabetic retinopathy grading performed after Clinical examination (slit lamp examination and OCT) correlates with that done virtually with Clarus fundus photos and OCT (A, B). The R3 grading includes both R3A and R3S. The Kappa coefficient shows there is fair agreement in diabetic retinopathy and maculopathy grading between slit lamp examination and virtual grading.

Table 3C summarises how the diabetic retinopathy grading done virtually by two graders correlates with each other. The Kappa coefficient shows there is almost perfect agreement in diabetic retinopathy between $\mathrm{G} 1$ and G2. Table 4 summarises the correlation between diabetic maculopathy grading from clinical examination and virtual grading (A, B) and between the two virtual graders (C).
Substantial agreement in diabetic maculopathy grading was observed between the two virtual graders.

\section{Discussion}

Widefield fundus camera imaging has evolved over the last 10 years to enable good quality wide angle fundus photos to be taken relatively easily and quickly and often without dilation. This technology has been used widely in virtual clinics where patients do not see a clinician face to face.

The Clarus widefield fundus camera has recently emerged to rival the Optos ultra-widefield fundus camera which has been the predominant widefield fundus camera at present. From a technical standpoint, the Optos fundus camera provides a single image of $200^{\circ}$ wide whereas the Clarus camera requires a montage of two separate fundus images to provide $200^{\circ}$ wide coverage. A field of view of $133^{\circ}$ of coverage is achievable with a single capture on the Clarus with an auto-montage of up to 6 photos being possible. In terms of colour, the Clarus fundus camera provides 
Table 3 (A) Correlation of diabetic retinopathy grading between slit lamp examination and virtual grader 1. (B) Correlation of diabetic retinopathy grading between slit lamp examination and virtual grader 2. (C) Correlation of diabetic retinopathy grading between virtual grader 1 and virtual grader 2 .

\begin{tabular}{|c|c|c|c|c|c|c|}
\hline \multicolumn{7}{|l|}{$\mathrm{A}$} \\
\hline & \multicolumn{6}{|c|}{ Virtual grading by G1 } \\
\hline & R0 & $\mathrm{R} 1$ & $\mathrm{R} 2$ & $\mathrm{R} 3$ & $\mathrm{U}$ & Total \\
\hline \multicolumn{7}{|c|}{ Grading from clinical examination } \\
\hline R0 & 13 & 5 & 0 & 0 & 1 & 19 \\
\hline R1 & 2 & 27 & 6 & 0 & 0 & 35 \\
\hline $\mathrm{R} 2$ & 0 & 8 & 16 & 0 & 3 & 27 \\
\hline R3 & 0 & 1 & 0 & 4 & 2 & 7 \\
\hline $\mathrm{U}$ & 0 & 0 & 0 & 0 & 2 & 2 \\
\hline \multicolumn{7}{|c|}{ Kappa $=0.561, \mathrm{SE}=0.069,95 \% \mathrm{CI} 0.426-0.696$} \\
\hline \multicolumn{7}{|l|}{$\mathrm{B}$} \\
\hline & \multicolumn{6}{|c|}{ Virtual grading by $\mathrm{G} 2$} \\
\hline & $\overline{\mathrm{R} 0}$ & $\mathrm{R} 1$ & $\mathrm{R} 2$ & R3 & $\mathrm{U}$ & Total \\
\hline \multicolumn{7}{|c|}{ Grading from clinical examination } \\
\hline R0 & 12 & 4 & 2 & 0 & 1 & 19 \\
\hline R1 & 2 & 25 & 8 & 0 & 0 & 35 \\
\hline $\mathrm{R} 2$ & 0 & 8 & 17 & 0 & 2 & 27 \\
\hline R3 & 0 & 1 & 0 & 6 & 0 & 7 \\
\hline $\mathrm{U}$ & 0 & 0 & 0 & 0 & 2 & 2 \\
\hline \multicolumn{7}{|c|}{ Kappa $=0.560, \mathrm{SE}=0.071,95 \%$ CI $0.420-0.699$} \\
\hline \multicolumn{7}{|l|}{$\overline{\mathrm{C}}$} \\
\hline & \multicolumn{6}{|c|}{ Virtual grading by $\mathrm{G} 2$} \\
\hline & $\overline{\mathrm{Ro}}$ & $\mathrm{R} 1$ & $\mathrm{R} 2$ & R3 & $\mathrm{U}$ & $\overline{\text { Total }}$ \\
\hline \multicolumn{7}{|c|}{ Virtual grading by G1 } \\
\hline R0 & 19 & 2 & 0 & 0 & 1 & 22 \\
\hline $\mathrm{R} 1$ & 0 & 40 & 4 & 0 & 0 & 44 \\
\hline $\mathrm{R} 2$ & 0 & 0 & 22 & 0 & 0 & 22 \\
\hline R3 & 0 & 0 & 0 & 4 & 0 & 4 \\
\hline $\mathrm{U}$ & 0 & 0 & 1 & 1 & 8 & 10 \\
\hline \multicolumn{7}{|c|}{ Каppa $=0.877$, SE $=0.039,95 \%$ CI $0.8-0.953$} \\
\hline
\end{tabular}

true colour images ${ }^{\mathrm{TM}}$ by using full spectrum white light. This is in contrast to the Optos Camera which provides a pseudocolour fundus image generated by combining only red and green light.

Hirano et al. compared the Clarus and Optos cameras to examine eyes with diabetic retinopathy using single images obtained with mydriasis, showing both systems are generally consistent in assessing diabetic retinopathy severity [13]. Figure 1(A) and (B) shows the $200^{\circ}$ Clarus fundus photos for a patient in our cohort with non proliferative diabetic retinopathy.
To the best of our knowledge, this is the first study evaluating the safety and validity of widefield Clarus fundus camera for the management of new referrals from DESP (DR and non-DR) in virtual clinics.

Our study of 51 patients showed that in about two-thirds of the new referrals from DESP included in the study, a diagnosis and management plan could be achieved using Clarus photos and macular OCT only, without the traditional outpatient clinic appointment and slit lamp examination. In particular, fair agreement of diabetic retinopathy grading between the virtual grading and slit lamp 
Table 4 (A) Correlation of diabetic maculopathy grading between slit lamp examination and virtual grader 1. (B) Correlation of diabetic maculopathy grading between slit lamp examination and virtual grader 2. (C) Correlation of diabetic maculopathy grading between virtual grader 1 and virtual grader 2 .

\begin{tabular}{|c|c|c|c|c|}
\hline \multicolumn{5}{|l|}{ A } \\
\hline & \multicolumn{4}{|c|}{ Virtual grading by G1 } \\
\hline & M0 & M1 & $\mathrm{U}$ & Total \\
\hline \multicolumn{5}{|c|}{ Grading from clinical examination } \\
\hline M0 & 57 & 6 & 4 & 67 \\
\hline M1 & 7 & 13 & 1 & 21 \\
\hline $\mathrm{U}$ & 1 & 0 & 1 & 2 \\
\hline \multicolumn{5}{|c|}{ Карpa $=0.487, \mathrm{SE}=0.097,95 \%$ CI $0.297-0.678$} \\
\hline \multicolumn{5}{|l|}{$\mathrm{B}$} \\
\hline & \multicolumn{4}{|c|}{ Virtual grading by $\mathrm{G} 2$} \\
\hline & M0 & M1 & $\mathrm{U}$ & Total \\
\hline \multicolumn{5}{|c|}{ Grading from clinical examination } \\
\hline M0 & 60 & 7 & 0 & 67 \\
\hline M1 & 6 & 14 & 1 & 21 \\
\hline $\mathrm{U}$ & 1 & 0 & 1 & 2 \\
\hline \multicolumn{5}{|c|}{ Kappa $=0.574, \mathrm{SE}=0.096,95 \%$ CI $0.385-0.762$} \\
\hline \multicolumn{5}{|l|}{$\mathrm{C}$} \\
\hline & \multicolumn{4}{|c|}{ Virtual grading by G2 } \\
\hline & M0 & M1 & $\mathrm{U}$ & Total \\
\hline \multicolumn{5}{|c|}{ Virtual grading by G1 } \\
\hline M0 & 68 & 3 & 0 & 71 \\
\hline M1 & 2 & 19 & 0 & 21 \\
\hline $\mathrm{U}$ & 5 & 1 & 4 & 10 \\
\hline
\end{tabular}

examination assessment were found in our study, whereas almost perfect agreement was observed between the two virtual graders.

There is variability in maculopathy grading between the virtual and clinical group even though OCT was used in both eyes. The variability is likely due to human variablity such as missing small exudates or judging how far away the exudates are from the fovea.

It should be noted that the discrepancy in diagnosis between face to face examination and virtual examination seen in our study did not alter the management plan for the vast majority of cases. Most differences were indeed noted regarding the severity of non proliferative diabetic retinopathy and this is not unusual between observers.

However, there was a case of proliferative diabetic retinopathy that was not identified by virtual assessment. Interestingly, the patient with missed retinal neovascularization was quite atypical in its presentation and he was referred by DESP because of the maculopathy in the fellow eye and not because of suspected neovascularisation. The eye with neovascularization was actually graded R1 by DESP and both virtual graders WL and GG and this was an early, low-risk neovascularization as judged by the doctor in clinic and a fluorescein angiogram was requested. The retinal neovascularization appeared like a blot haemorrhage on the Clarus photo, but the peripheral retina view was slightly fuzzy (Fig. 1d). There were hardly any retinal haemorrhages elsewhere in the retina and this was a small atypical vessel. The other eye of the same patient had treated inactive proliferative diabetic retinopathy (Fig. 1c). Fluorescein angiography is an immediately available option for the doctor carrying out the clinical exam and this availability would make it a more requested option than for those reviewing images alone.

We propose having a low threshold of bringing back patients for slit lamp examination if the peripheral retina 
Fig. 1 Figure 1 shows examples of Clarus fundus photos for 2 patients. A and $\mathbf{B}$ are montage of 2 photos combining to give a field of $200^{\circ}$. They are from a patient with non proliferative diabetic retinopathy. $\mathbf{C}$ and $\mathbf{D}$ are single photos of $133^{\circ}$ wide from a patient with proliferative diabetic retinopathy. The inferior blot haemorrhage seen in $\mathbf{D}$ was shown to be neovasularisation on fluorescein angiogram.
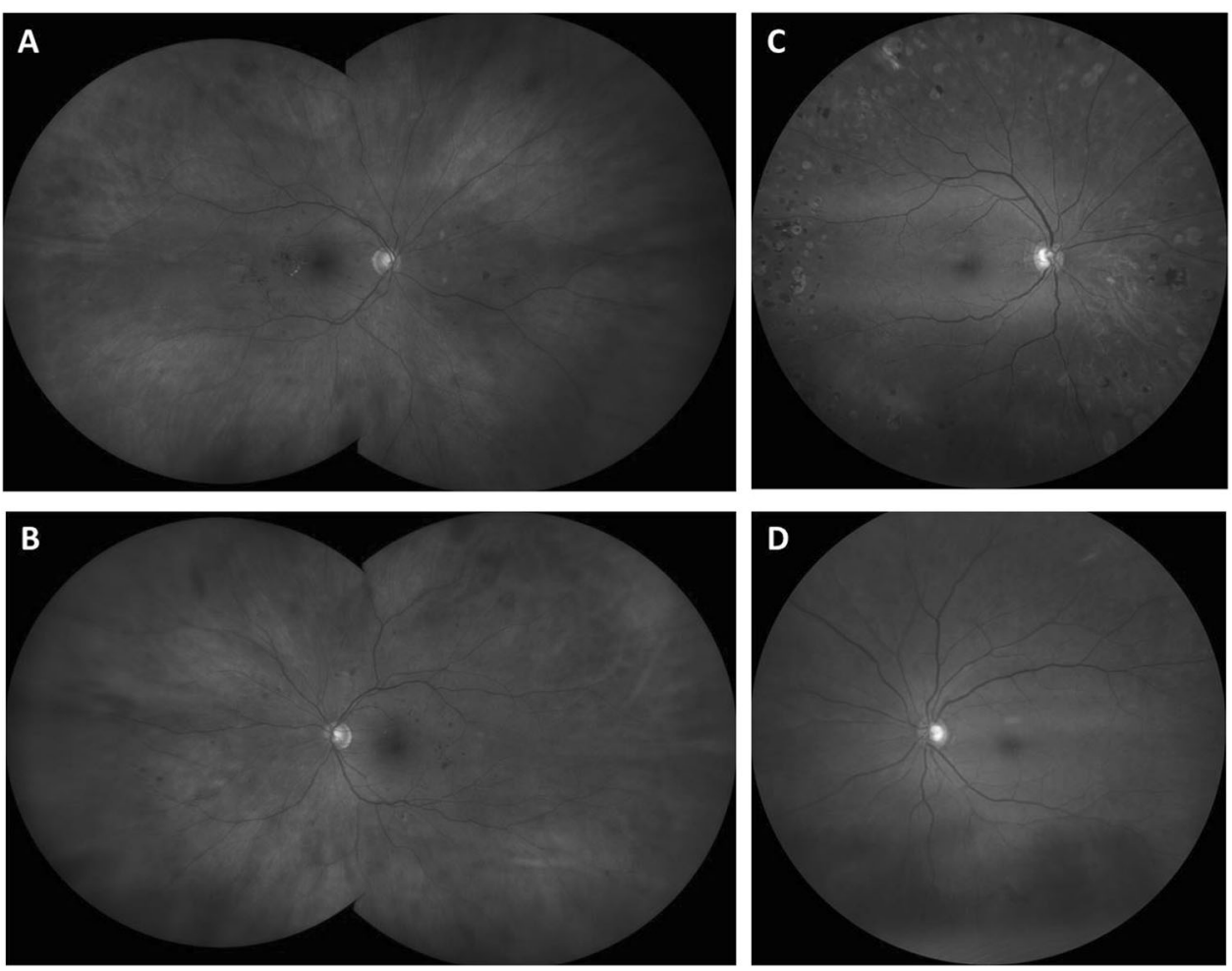

view on the Clarus photo is hazy with suspicious retinal haemorrhage or if the other eye has proliferative disease.

Manjunath et al. showed that Optos widefield fundus photos could fail to show retina neovascularization diagnosed on clinical examination [11]. They reported the use of Optos imaging had $73 \%$ sensitivity in detecting proliferative diabetic retinopathy. Our study only had two eyes with active proliferative retinopathy therefore calculating reliable sensitivities for detection of proliferative disease was beyond the scope of our study.

It should be noted that virtual assessment has a few limitations and may not be a viable option in all cases. For instance, in our study although both graders were able to identify photic maculopathy from the images they were unable to confirm this, as this is done with a direct question to the patient It should be noted that graders were blinded to the outcomes of face-to-face review and unaware of the patient's history. If the virtual assessment was combined with telephone consultation to take a history, then the diagnosis of photic maculopathy could have potentially been made as the history of exposure is usually revealed through discussion with the patient.

If a patient was assessed virtually and felt to need treatment such as anti-VEGF or further blood tests in retinal vein occlusion, this could be conveyed to the patient and GP by telephone call and letter, with the advantage that patients would then have time to consider the options for therapy (or not), discuss this at home and write down any questions prior to consultation in the medical retina clinic. In our study, the patient with choroidal naevus was assessed to need further face to face examination or further scans but potentially they could be kept in virtual clinics if an OCT scan can be carried out over the naevus.

The main presentation where virtual clinics would be unsuitable would be when the fundus view is hazy, as frequently observed in the presence of cataracts. Furthermore, some patients may have reduced vision that cannot be explained by retinal imaging. If these patients can have adequate anterior segment and optic disc imaging, they could potentially still have virtual assessment a utility similar to slit lamp examination.

Lastly, it may be challenging to take a fundus photograph in some patients. Obtaining fundus pictures in patients who are unable to keep their head still might be a challenge, making this subgroup unsuitable for virtual imaging. Although such patients could equally be difficult to examine on a slit lamp, the ophthalmologist in clinic would have the option of other means of visualising the fundus.

On the other hand, the use of virtual clinics has become more important in light of the COVID-19 pandemic as virtual clinics might reduce the level and duration of close contact between people. Reduced waiting time in virtual clinics may also prevent crowding in the waiting room in order to allow adequate social distancing for those who do need to be seen in the clinic. This is especially important as our patients are often elderly or have diabetes and quite often other comorbidities that increase their susceptibility to COVID-19. There will also be a huge backlog of patients to 
be seen after the lockdown period has ended. Virtual clinics could indeed help to meet the increased demand for hospital eye clinic appointments as Lee et al. showed that twice as many patients can be seen in virtual clinics compared to slit lamp examination clinics in the same time period [4]. There is also the potential for fundus photographs and OCT scans to be carried out in the community, close to the patients' home, thus minimising their travel time and the images would then be reviewed by ophthalmologists remotely and a virtual consultation could be arranged.

\section{Conclusion}

Virtual assessment with Clarus widefield fundus imaging and macular OCT was found to be a valid and safe option for the assessment of new referrals from DESP, allowing two-thirds of referrals to be safely diagnosed and managed with no need to face-to-face review. Caution is recommended for cases with poor quality of imaging, unexplained visual decrease or findings suspicious of high-risk retinal pathology for whom slit lamp review might be required. A thorough triage of referrals is advisable to identify patients suitable for virtualisation.

\section{Summary}

\section{What was known before}

- Widefield fundus imaging can be as good as slit lamp fundus examination in picking pathologies.

- Widefield fundus imaging with Optos camera has been used widely in virtual medical retina clinics.

\section{What this study adds}

- Widefield fundus imaging with the Clarus camera is useful in diagnosing retina pathology and could be used in virtual clinics.

- Widefield fundus imaging with the Clarus camera combined with OCT can be used to manage $2 / 3$ of new referrals from diabetic retinopathy screening with a good safety profile.

Funding Mr Rajendram has received speaker fees from Zeiss.

\section{Compliance with ethical standards}

Conflict of interest The authors declare that they have no conflict of interest.

Publisher's note Springer Nature remains neutral with regard to jurisdictional claims in published maps and institutional affiliations.

\section{References}

1. Scanlon PH. The English National Screening Programme for diabetic retinopathy 2003-2016. Acta Diabetol. 2017;54:515-25.

2. Wild S, Roglic G, Green A, Sicree R, King H. Global prevalence of diabetes: estimates for the year 2000 and projections for 2030 . Diabetes Care. 2004;27:1047-53.

3. rcophth-The-Way-Forward-AMD-Summary-300117-1.pdf [Internet]. https://rcophth.wpengine.com/wp-content/uploads/2015/10/ RCOphth-The-Way-Forward-AMD-Summary-300117-1.pdf.

4. Kern C, Kortuem K, Hamilton R, Fasolo S, Cai Y, Balaskas K. et al. Clinical outcomes of a hospital-based teleophthalmology service: what happens to patients in a virtual clinic? Ophthalmol Retina. 2019;3:422-8.

5. Kortuem K, Fasler K, Charnley A, Khambati H, Fasolo S, Katz M, et al. Implementation of medical retina virtual clinics in a tertiary eye care referral centre. Br J Ophthalmol. 2018;102:1391-5.

6. Lee JX, Manjunath V, Talks SJ. Expanding the role of medical retina virtual clinics using multimodal ultra-widefield and optical coherence tomography imaging. Clin Ophthalmol. 2018;12:2337-45.

7. Hinkle JW, Flynn HW, Banta JT, Vanner EA. Patients presenting emergently with proliferative diabetic retinopathy: follow-up and factors associated with compliance. Retin Philos. 2020;40:928-35.

8. Ashraf M, Shokrollahi S, Salongcay RP, Aiello LP, Silva PS. Diabetic retinopathy and ultrawide field imaging. Semin Ophthalmol. 2020;35:56-65.

9. Aiello LP, Odia I, Glassman AR, Melia M, Jampol LM, Bressler $\mathrm{NM}$, et al. Comparison of early treatment diabetic retinopathy study standard 7-field imaging with ultrawide-field imaging for determining severity of diabetic retinopathy. JAMA Ophthalmol 2019;137:65-73.

10. Purbrick RMJ, Izadi S, Gupta A, Chong NV. Comparison of Optomap ultrawide-field imaging versus slit-lamp biomicroscopy for assessment of diabetic retinopathy in a real-life clinic. Clin Ophthalmol. 2014;8:1413-7.

11. Manjunath V, Papastavrou V, Steel DHW, Menon G, Taylor R, Peto $\mathrm{T}$, et al. Wide-field imaging and OCT vs clinical evaluation of patients referred from diabetic retinopathy screening. Eye 2015;29:416-23.

12. Landis JR, Koch GG. The measurement of observer agreement for categorical data. Biometrics 1977;33:159-74.

13. Hirano T, Imai A, Kasamatsu H, Kakihara S, Toriyama Y, Murata T. Assessment of diabetic retinopathy using two ultra-wide-field fundus imaging systems, the Clarus ${ }^{\circledR}$ and Optos ${ }^{\mathrm{TM}}$ systems. BMC Ophthalmol. 2018;18:332. 